\title{
Cinquante ans de réalisations : la Société des Missions-Étrangères
}

\section{Claude Guillet}

Volume 38, 1971

La genèse de la Société des Missions-Étrangères de la province de Québec

URI : https://id.erudit.org/iderudit/1007265ar

DOI : https://doi.org/10.7202/1007265ar

Aller au sommaire du numéro

Éditeur(s)

Les Éditions Historia Ecclesiæ Catholicæ Canadensis Inc.

ISSN

0318-6172 (imprimé)

1927-7067 (numérique)

Découvrir la revue

Citer cet article

Guillet, C. (1971). Cinquante ans de réalisations : la Société des

Missions-Étrangères. Sessions d'étude - Société canadienne d'histoire de l'Église catholique, 38, 55-69. https://doi.org/10.7202/1007265ar

Tous droits réservés @ C Les Éditions Historia Ecclesiæ Catholicæ Canadensis Inc., 1972
Ce document est protégé par la loi sur le droit d'auteur. L'utilisation des services d’Érudit (y compris la reproduction) est assujettie à sa politique d'utilisation que vous pouvez consulter en ligne.

https://apropos.erudit.org/fr/usagers/politique-dutilisation/ 


\section{Cinquante ans de réalisations: La Société des Missions-Étrangères}

Un mouvement général nouveau surgit dans l'Église, à la fin du $\mathrm{XIX}^{\bullet}$ siècle et au début du $\mathrm{XX}^{\circ}$. Durant cette période naissent en Italie, en Suisse, en Irlande, aux États-Unis, au Canada anglais, des instituts de prêtres séculiers qui désirent se consacrer à l'activité mission. naire dans des pays non chrétiens.

C'est dans ce courant que s'insère la fondation de la Société des Mis. sions-Êtrangères du Québec en 1921. Et l'originalité de cette fondation se trouve dans le fait que les évêques de la province de Québec en ont pris l'initiative et assuré le développement ultérieur.

Le rôle de cette Société dans l'effort missionnaire global canadien doit être perçu en fonction de l'originalité de sa fondation. En effet, les 400 membres qu'elle a comptés depuis les débuts en 1921 ne forment qu'une minorité parmi les quelque 7000 missionnaires canadiens, hommes et femmes. Ce n'est donc pas de ce côté-là qu'il faut chercher la contribution spéciale de la Société à l'effort missionnaire canadien, même si elle n'est pas négligeable.

Son rôle spécial a été de représenter, sous la poussée des Évêques, un secteur de l'Église canadienne dans les pays étrangers, soit le clergé local séculier. Celui-ci avait déjà entrepris depuis longtemps une vérita. ble activité missionnaire en Amérique du Nord, mais n'avait pu le faire en dehors du Continent. C'est cette possibilité nouvelle que représenta la fondation de la Nouvelle Société, qui devenait aussi un peu un instrument officiel de l'épiscopat de langue française.

Essayer de donner une idée concrète et détaillée des réalisations de la Société des Missions-Étrangères depuis ses débuts jusqu'à aujourd'hui dans les territoires qu'on lui a confiés serait trop long. D'abord parce que le nombre de personnes à présenter et la masse de faits à situer et à expliquer deviendraient beaucoup trop imposants. Ensuite, parce que je crois plus profitable de me situer à un niveau de considération un peu plus général. Je voudrais essayer d'analyser à grands traits les caractéristiques des éléments humains qui ont été mis en présence par le fait même de l'activité de la Société des Missions-Étrangères, et essayer de saisir le résultat de cette rencontre.

Nous essaierons donc de répondre, quoique trop rapidement et trop superficiellement, à trois questions : 
Quel genre de missionnaires la Société des Missions-Étrangères a-t-elle envoyés, durant la majeure partie de ces années, dans ses territoires de missions? Quelles étaient les principales caractéristiques des pays dans lesquels la Société envoyait ses sujets ?

Quel genre d'action missionnaire est-il résulté de la rencontre entre ces deux facteurs?

La Société des Missions-Étrangères est née de la volonté des chefs de l'Église canadienne de donner au clergé et au peuple canadiens-français un rôle officiel dans l'œuvre d'évangélisation de l'Église. Mais en dehors même de cette volonté explicite, il était inévitable que les caractéristiques de leur milieu d'origine marquent ces missionnaires.

Quelles étaient ces caractéristiques?

Dans les années 30, les Canadiens français étaient au nombre d'à peu près 3 millions. C'était un petit groupe culturel perdu dans un contexte anglo-saxon et dominé économiquement par les Anglais, les Canadiens anglais et les Américains. En général, c'était une collectivité pauvre, d'autant plus qu'une crise économique grave commençait et qui devait durer une dizaine d'années. La mentalité était encore sinon à dominante rurale, au moins nettement provinciale. Un milieu homogène, où les traditions culturelles étaient fortes et préservaient la cohésion sociale d'une population simple, saine, et assez peu instruite. Cette cohésion était due entre autres à un Église catholique omniprésente, forte, active, influente.

Cette influence de l'Église, on pouvait la remarquer dans l'ordre politique. Si on ne peut pas parler d'union de l'Église et de l'État, on peut dire qu'il existait la plupart du temps un accord entre les deux sphères de pouvoir. Leur double présence globale sur une population limitée imposait dans la pratique souvent une entente. Et de plus, l'origine d'un même milieu homogène, la même formation dans les mêmes collèges classiques et des contacts facilement maintenus entre les dirigeants des deux pouvoirs facilitaient chez eux une vision des choses souvent identiques.

L'Église exerçait aussi une position dominante dans les domaines de l'éducation, de la santé et des loisirs. Et cette influence, l'Église la devait surtout à la présence et à l'action de ses prêtres, de ses religieux et religieuses. En résumé, l'Église était une grande force religieuse, culturelle, sociale et politique.

Voilà pour le milieu général d'où sortaient les membres des Missions-Étrangères. Un peuple dominé, sans arrogance culturelle, ce qui devait favoriser une adaptation plus facile à des peuples et à des cultures différentes.

Quelle sorte d'hommes étaient ces missionnaires au moment où ils se lançaient sur les routes du monde? Ils ressemblaient fortement aux 
prêtres d'ici. Ils étaient membres d'un groupe conscient de l'importance de son rôle ecclésial et social, un groupe fermement constitué, assez homogène, sûr de la direction de son action, et habitué à l'exercice d'une autorité souvent paternaliste.

Une formation à peu près identique, formation stricte, sévère, en milieu assez fermé inculquait à ces hommes des comportements, des attitudes, des modes d'être, et de relations qui les classaient clairement dans le groupe social du clergé. Leur formation intellectuelle était nettement ecclésiastique : philosophie occidentale scolastique, un peu abstraite et désincarnée, théologie intellectualiste un peu sèche aussi, pas toujours près de la vie. Leur formation morale cependant était solide. Elle en faisait des hommes énergiques, travaillants, plus portés à une vie d'action concrète sur les choses et les hommes, qu'à une vie intellectuelle intense et à une étude approfondie de leur milieu.

Ils reflétaient aussi dans leur manière d'être le type de religion qu'était alors le catholicisme canadien-français. Une religion fortement structurée, hiérarchique, qui, avec énergie, se défendait des influences et des attaques que le monde moderne lui infligeait, monde dont elle avait de la difficulté à analyser les grandes orientations politiques, sociales et économiques. Une religion sur la défensive au plan intellectuel, et en état d'offensive sur le plan de l'action sociale et missionnaire. Une Église qui encadrait ses fidèles dans des cadres rigides, des lois strictes et souvent dans des attitudes de retrait ou d'isolement.

Une Église qui transmettait certes avec vigueur et courage, le contenu de sa foi résumé dans le Credo mais qui produisait très souvent un type de chrétien pour qui les rites sacramentels et les pratiques dévotionnelles avaient tendance à prendre une importance trop exclusive et chez qui on cherchait à développer plus les vertus individuelles que les vertus d'engagement social.

Le catholicisme canadien-français était donc un système global où la foi proprement dite était insérée dans une religion de type plutôt individualiste, ritualiste, n'échappant pas toujours à une mentalité magique, préoccupée plus par le salut de l'âme de l'individu que par le souci de constituer des communautés chrétiennes bien vivantes.

En gros, ce que nous venons de dire s'applique non seulement au catholicisme canadien-français mais aussi avec des variantes mineures au catholicisme universel. Celui-ci était vraiment une foi universelle mais s'exprimant dans une religion dont les formes et la mentalité étaient occidentales. Le fait n'est pas sans importance quand il s'agit d'apprécier toute une période de l'action missionnaire de l'Église universelle.

La deuxième question que nous avons posée porte sur les principales conditions des pays dans lesquels la Société des Missions-Étrangères a 
envoyé ses membres? Nous répondrons aussi immédiatement à la troisième question sur le genre d'action missionnaire qui est résulté de ces conditions.

\section{La Chine}

Pays immense, de très vieille civilisation, la Chine au XIX ${ }^{\bullet}$ siècle était endormie. D'autres allaient la réveiller et brutalement l'obliger à se rendre compte que son système économique, culturel et politique ne pouvait la protéger contre les assauts successifs de l'impérialisme occidental, russe et japonais. Pendant près de cent ans, la Chine ne sera plus vraiment maître de son histoire, même si un gouvernement national chinois est maintenu. Elle se trouve brusquement confrontée au monde moderne. Il lui faut apprendre durement à travers des humiliations successives les rudiments de ce monde nouveau : science et technique, économie et commerce capitalistes, armements et guerres modernes.

C'est à ce moment-là que l'Église, sur les pas des puissances occidentales, pénètre de nouveau en Chine. Situation complexe, délicate et dangereuse.

Certes, les puissances occidentales permettent au christianisme de pénétrer en Chine en lui faisant accorder la liberté d'action et en lui promettant et accordant sa protection. Mais il est dangereux pour une religion d'essayer de pénétrer ainsi, comme par effraction, une vieille culture comme celle de la Chine. Cela peut donner lieu à un phénomène de rejet lorsque le pays recouvre sa pleine liberté d'action, sa fierté et sa dignité. Sans plus d'explications, disons que c'est ce qui semble être arrivé.

Ainsi, dans les circonstances où la Chine a de nouveau rencontré le christianisme, elle était dans un état d'infériorité, d'impuissance et ses traditions culturelles étaient remises en question. Conditions qui ne favorisaient pas de la part d'étrangers, des efforts sérieux et profonds pour une adaptation du christianisme au milieu culturel de la Chine. C'est là une des raisons pour lesquelles ce que l'Église a transmis à la Chine est un christianisme occidental non seulement dans son esprit mais même dans la langue et ses rites.

Lorsque le 17 octobre 1925, les 2 premiers P.M.E. arrivaient en Chine, c'était donc dans un pays profondément troublé par la présence des étrangers et par les divisions internes : provinces autonomes, développement des forces communistes, extension du banditisme, et bientôt invasion de la Mandchourie et du Jéhol par les Japonais.

Malgré ces circonstances politiques, sociales et économiques difficiles, le travail que vont réaliser les P.M.E. dans leur première mission sera vraiment quelque chose d'appréciable, surtout si on considère la pauvreté des moyens à leur disposition. 
Au moment de la déclaration de la guerre entre les puissances occidentales et le Japon, soit 15 ans après le début de la mission, en 1941, il y avait dans le diocèse de Szepingkai et dans la préfecture apostolique de Lintung, 50 prêtres canadiens: 45 P.M.E. et 5 Clercs de Saint-Viateur, et 12 Frères de Saint-Viateur. Les Sœurs de l'Immaculée-Conception étaient au nombre de 47, les Sœurs chinoises de Notre-Dame-du-Rosaire au nombre de 25, et 5 Sœurs Antoniennes de Marie.

Ce personnel était responsable de 25000 chrétiens dans une population de plus de 3 millions d'habitants. Les Canadiens occupaient 20 postes paroissiaux avec résident et visitaient aussi 40 dessertes.

Gagner au Christ une telle population n'était pas chose facile, si on tient compte des dures conditions matérielles dans lesquelles ce travail devait se faire: pauvreté du pays, moyens de communications lents, difficiles et souvent dangereux. De plus les contacts avec une population simple, peu instruite, mais très différente culturellement, et l'apprentissage long d'une langue difficile demandaient beaucoup d'efforts et de patience.

Les missionnaires se sont efforcés d'instituer ce qui pour eux devaient être les structures de base de la mission. D'abord les paroisses et les dessertes sont établies parmi les petits groupes de chrétiens qu'ils trouvent sur les lieux à leur arrivée : 2673 Chrétiens. Ils organisent ensuite un système de catéchuménats pour adultes chinois avec une école pour catéchistes à Szepingkai. On pense ensuite aux écoles : 6 écoles primaires : 1700 élèves et une école supérieure commerciale avec 430 étudiants, toutes approuvées par le gouvernement; et puis près de 80 écoles de prières, fréquentées par plus de 1500 enfants chrétiens où on enseigne le catéchisme, les prières et les premières notions des lettres chinoises.

Parce que le problème du clergé indigène est la première préoccupation des Pères canadiens, on pense très tôt, en 1934. à Szepingkai et en 1938 à Lintung, à construire des petits séminaires. Les autres communautés religieuses s'efforcent aussi de se trouver sur les lieux des vocations. Malgré le peu d'années de travail, le résultat sera très positif : une quinzaine de prêtres séculiers chinois et de nombreuses vocations pour les autres communautés.

Leur compassion chrétienne amène naturellement les missionnaires à s'occuper des malheurs concrets de leur population. C'est pourquoi avec l'indispensable coopération des religieuses, ils organisent 24 dispensaires de soins médicaux qui donneront plus de 300000 traitements annuellement, 4 orphelinats, et 13 hospices pour vieillards.

C'est par toute cette activité que les missionnaires étaient en train de faire de la mission de Mandchourie une réussite qui promettait encore 
plus pour l'avenir. C'est par elle que, malgré les limites que nous avons marquées, ils gagnaient les populations parmi lesquelles ils travaillaient. Mais surtout c'est leur simplicité, leur absence de mentalité colonialiste et leur amour réel de leurs fidèles qu'ils ont conquis leur affection.

Mais ces promesses d'avenir seront coupées par la brutalité des grands courants historiques de notre époque. C'est d'abord la guerre de 1935-1945 durant laquelle le travail sera presque totalement arrêté parce que tous les missionnaires sont incarcérés à Szepingkai durant 4 ans par les Japonais.

Et cette guerre n'est pas aussitôt terminée, qu'une autre commence qui rend de plus en plus difficile le travail pour les missionnaires de moins en moins nombreux : c'est la guerre civile chinoise entre nationalistes de Tchang Kai-Shek et les communistes de Mao Tse-toung qui se terminera en 1949 par la victoire de celui-ci.

Ce qui reste de la jeune Église de Szepingkai est alors balayé par ce terrible ouragan, comme les autres Églises de Chine. Entre temps, les missionnaires canadiens auront démontré leur attachement à la foi de l'Église, à leur peuple de Mandchourie par le courage avec lequel ils ont supporté les souffrances causées par ces événements.

\section{Les Iles Philippines}

En 1937, à la demande de Rome et de $\mathrm{M}^{\mathrm{gr}}$ Luis del Rosario, évêque de Zamboanga dans l'île Mindanao aux Philippines, la Société des Missions-Étrangères acceptait une deuxième mission : la province de Davao qui faisait alors partie du diocèse de Zamboanga.

Les Philippines, pendant trois siècles et demi, colonie de l'Espagne qui leur a apporté la culture espagnole et la foi chrétienne, étaient depuis 1898 sous la domination américaine. Ces nouveaux colonisateurs accentuèrent le processus d'unification politique en dotant le pays d'un régime démocratique et d'un système d'éducation publique neutre unifié par la langue anglaise.

Sur une population alors de 13 millions, près de $85 \%$ étaient catholiques. Seul pays catholique de l'Orient, mais d'un catholicisme bien anémique. L'ancien clergé espagnol ne s'était guère soucié d'y former un clergé national. Ainsi au départ des Espagnols, à la fin du XIX ${ }^{\bullet}$ siècle, il restait peu de pasteurs pour s'occuper du peuple chrétien. On ne pouvait alors s'étonner de l'ignorance religieuse dans l'archipel et du très grand nombre de non-pratiquants. En fait, on se trouvait en face d'un christianisme trop souvent superficiel, d'un système religieux de pratiques et de cérémonies sans influence profonde sur la vie. Beaucoup d'observateurs pensent que la conversion des régions rurales au catholicisme a surtout consisté en l'incorporation de certains symboles chrétiens dans un système antérieur de croyances animistes, ce système étant ainsi élargi 
mais non modifié radicalement. Cette situation de mélange de christianisme et d'animisme a abouti à ce qu'on appelle aux Philippines " split level christianity ..

Beaucoup de progrès ont été réalisés depuis les jours d'octobre 1937 où les cinq premiers prêtres canadiens débarquaient à Davao. Cette région de 155000 catholiques était desservie par seulement 7 prêtres déjà âgés.

Sauf durant le conflit mondial dont les Philippines ont été l'un des principaux théâtres en Extrême-Orient et qui a interrompu le travail pendant 4 ans, le nombre de missionnaires canadiens augmentait d'année en année. Mais la population croîssait à un rythme encore plus élevé, surtout après la guerre, par l'arrivee d'innombrables vagues d'immigrants, venus principalement des provinces centrales du pays Avec cette immigration et le taux élevé de natalité, la population de ce territoire, de 155000 en 1937, passa à 1510000 environ en 1970.

Mais déjà en 1962, les P.M.E., débordés, avaient fait appel aux missionnaires américains de Maryknoll qui prirent en charge la partie nord du diocèse, soit environ le tiers en population et la moitié en superficie. En 1970, pour une population de 837000 catholiques, il y avait 105 prêtres dont 75 P.M.E. dans la région de Davao.

Ces populations si nombreuses en majorité catholiques réclamaient le baptême pour leurs enfants. La plupart exigeait également de leur clergé qu'il bénisse leur mariage et qu'il fasse des prières spéciales pour leurs défunts, les « responsos ». Au moins une fois par année, le prêtre devait aussi visiter dans des conditions difficiles, souvent jusqu'à une centaine de villages éloignés. Ces seules activités de célébration des sacrements, avec les tâches connexes de la construction de lieux de culte, l'administration matérielle de la paroisse, etc., occupaient déjà la majeure partie du temps des missionnaires, en nombre totalement insuffisant pour toutes les tâches d’Église.

Sur le plan de l'éducation, les missionnaires ont établi, dans les paroisses, 10 écoles primaires, 28 écoles secondaires catholiques et, dans les centres plus importants, 13 écoles d'enseignement supérieur (préuniversitaire). Ces écoles étaient un lieu privilégié d'instruction chrétienne et, surtout au niveau secondaire et supérieur, un service à la population sur le plan académique, puisque l'État n'y pourvoyait pas encore.

Ces tâches d'enseignement dépassaient les possibilités des P.M.E. qui, au fur et à mesure des besoins, firent appel à des communautés enseignantes, dont les Jésuites de la province américaine, les Sœurs de l'Immaculée-Conception de Montréal, les Sœurs de l'Assomption du Nouveau-Brunswick, les Sœurs de la Présentation de la Nouvelle-Angle- 
terre et les Frères du Sacré-Cour de la province d'Ottawa. Pour leur part, les Sœurs Dominicaines de Québec venaient, par la fondation d'hôpitaux, prêter main-forte à l'unique hôpital public de Davao.

En même temps, les missionnaires organisèrent aussi l'Action catholique, surtout chez les jeunes, étudiants et ouvriers et divers mouvements apostoliques: Barangay, Légion de Marie, "cursillos de cristiandad ".

Mais une Église n'est pas adulte tant qu'elle n'a pas ses propres pasteurs. C'est pourquoi, dès le début, les P.M.E. se sont préoccupés de la formation d'un clergé local. En 1947, en érigea un petit séminaire pour recevoir les jeunes qui présentaient des aptitudes sérieuses au sacerdoce : en 1970, 141 séminaristes y étudiaient.

En 1963, $\mathbf{M}^{\mathrm{gr}}$ Thibault songeait à construire un grand séminaire diocésain. Le Saint-Siège demanda alors qu'il soit plutôt au service des 12 diocèses de l'île de Mindanao. En cinq ans, ce grand séminaire a déjà donné 17 prêtres à cette région sud du pays. Actuellement, il compte 22 étudiants en théologie et 104, en philosophie dont 26 pour Davao.

Davao compte maintenant 14 prêtres philippins. Depuis trois ans, il avait un évêque-coadjuteur philippin, $M^{\text {gr }}$ Juan Nilmar. Et en 1970, Davao, de territoire de mission qu'il était au début, devenait archidiocèse. Mais si cette dernière proclamation est un point d'arrivée, elle est davantage un point de départ. Car des orientations nouvelles, suggérées par Vatican II et la situation du pays, viennent s'ajouter à l'augmentation des tâches, issues du travail même des P.M.E. jusqu'à ce jour : entre autres, approfondissement du travail d'évangélisation et intensification des activités dans le domaine social.

\section{Le Japon}

En 1945, le Japon subit la catastrophe de la défaite. Il assiste à l'écroulement de son vieil édifice politique et à la perte de l'Empire qu'il s'était progressivement acquis depuis la fin du XIX ${ }^{\bullet}$ siècle. Dans un pays ravagé, le peuple désorienté et découragé se voit pour la première fois de son histoire soumis à un envahisseur qui va cependant l'aider à se relever.

C'est le moment que l'Église choisit pour faire des efforts spéciaux pour, de nouveau, là aussi, essayer de pénétrer de l'Évangile du Christ ce peuple mystérieux et séduisant, le premier peuple de couleur à avoir assimilé avec une telle rapidité les sciences et les techniques occidentales. Pays industrialisé, peuple instruit, nation d'une culture ancienne et autonome qui se renouvelle, se transforme, se cherche sous l'impact des courants internationaux, c'est à tout cela que l'Église doit faire face. Et la tâche n'est pas facile. 
C'est pourquoi l'Église au Japon est une Église en recherche. Il lui faut réfléchir sur le fait chrétien dans un pays moderne non chrétien et sur les méthodes d'apostolat propres à un tel contexte. C'est une Église qui expérimente beaucoup, qui ouvre une foule de voies dans l'apostolat, qui multiplie, pour faire connaître le Christ, ses activités dans l'éducation, dans le domaine social et pastoral.

En 1948, à la demande de Rome, la Société des MissionsÉtrangères envoie dans ce pays ses premiers missionnaires, qui s'installent à Tokyo et bientôt dans le Nord du Japon dans la préfecture d'Ao. mori qui fait partie du diocèse de Sendai et enfin dans le diocèse de Yokohama en 1960.

La première tâche pour tous les missionnaires est l'apprentissage long et difficile de la langue japonaise qui demande plusieurs années d'études. Une fois cet instrument acquis, c'est le travail de base en paroisse. Les missionnaires au nombre de 14 en 1951 dirigent 6 paroisses et 8 dessertes, tandis qu'en 1961, parvenus au nombre de 33, ils ont pris charge de 13 paroisses et de 7 dessertes. Les catholiques de Sendai en 1950 étaient au nombre de 974, en 1970, de 12 541.

Le plus difficile au Japon est le contact avec les gens. Aussi faut-il trouver des moyens de multiplier ces contacts. Les écoles d'abord : 13 écoles maternelles tenues par les P.M.E., 3 écoles élémentaires et 8 écoles secondaires et supérieures, tenues par plus de 150 religieuses canadiennes, toutes sont des occasions précieuses pour les enfants et leurs parents d'une connaissance plus concrète de l'Église et de l'Évangile. On s'efforce aussi de travailler dans les écoles publiques ou les universités comme professeurs de morale, de langue, de littérature ou de Bible. L'utilisation des moyens de communications, imprimé, radio, télévision est une nécessité. On fonde des œuvres comme pensions ou centres pour étudiants universitaires, hospices, orphelinats, léproserie pour une insertion concrète du missionnaire dans les besoins du milieu.

Le résultat de cette activité diversifiée au Japon se manifeste dans une sympathie pour le christianisme en hausse réelle : 3 millions de Japonais se reconnaissent d'esprit chrétien alors que le Japon ne compte que 700000 baptisés dont 350000 catholiques.

\section{L'Amérique latine}

Dans les années cinquante, une préoccupation se développe en Europe et en Amérique du Nord et du Sud, celle de la situation de l'Église en Amérique latine, préoccupation qui naît d'une vive prise de conscience : 200 millions de catholiques soit $34 \%$ de la population catholique du globe; population prévisible dans 50 ans : 600 millions. Pour desservir cette population, à peine 35000 prêtres soit $91 / 2 \%$ du total des prêtres dans l'Église; un catholicisme de peu de vitalité, d'une influence 
bien relative sur les milieux sociaux. Et pour aggraver ces maux et en retarder la guérison, les catholiques de ces diverses républiques sont trop repliés sur leur individualisme, manquent d'esprit de coopération, et l'Église elle-même est trop liée à la classe possédante aristocrate, d'une foi souvent plus que tiède et dépourvue de clairvoyance.

Durant des années, les différentes Églises allaient intensifier leurs activités dans ce continent afin de se préparer à faire face aux immenses problèmes politiques, sociaux, économiques et religieux qui se posaient alors, qu'on savait ne plus pouvoir éluder, et qui allaient exiger des solutions neuves et courageuses.

Cuba

C'est dans ce contexte général que la Société des MissionsÉtrangères allait insérer son action. D'abord orientée vers l'Orient : Chine, Japon et Philippines, elle se tourne maintenant vers l'Occident, vers l'Amérique latine. D'ailleurs, elle avait posé, dès 1942, un premier geste dans ce sens au moment où ses territoires de Chine et des Philippines étaient impliqués dans la guerre. Cette année-là, elle accepte un territoire à Cuba : l'île des Pins, d'une population de 6000 habitants. Mais 2 ans plus tard, les Pères canadiens vont plutôt travailler sur la grande île, dans les deux diocèses de la Havane et de Matanzas où on leur confie 2 vicariats forains avec une population de 150000 habitants et 13 paroisses.

Comme aux Philippines, la grande majorité de la population est baptisée, mais ignorante de sa religion et éloignée de l'Église. Par tous les moyens, les P.M.E. cherchent à aller chercher les chrétiens pour les ramener à l'Église : visites de familles, terrains de jeux, fondations d'écoles pour toutes les classes de la société, ouverture de centres catéchistiques, prédication de petites missions, travail sur la famille, cours de préparation au mariage, action catholique, création d'une cité étudiante à Colon avec un collège classique pour garçons, un collège pour filles et un séminaire diocésain qui ne craindra pas de recevoir, ce qui est nouveau à Cuba, des jeunes gens plus âgés qui deviendront des prêtres de première qualité. Travail social aussi par les Caisses populaires et la création d'une école de métier.

C'était une Église en plein progrès et en pleine expansion. En 1961, 4.1 P.M.E. dans 16 paroisses avec 22 églises et 20 dessertes avec chapelles pour une population de 210000 habitants : 22 écoles primaires, 1 école secondaire, 1 école commerciale, un petit séminaire, sans compter les associations d'Action catholique et les activités d'enseignement catéchistique.

Mais cette même année-là, la situation allait changer à Cuba. Le gouvernement de Castro se radicalisa et l'Église se retrouva dans la si- 
tuation difficile où son action se vit limitée au seul domaine de la liturgie et de l'enseignement religieux à l'église, situation rendue plus dure par la perte d'une bonne partie de son personnel sacerdotal et religieux. Une vingtaine de P.M.E. continuent cependant leur travail dans ces nouvelles conditions et s'efforcent de trouver pour une Église affaiblie et minoritaire un mode de présence et d'action nouvelles par la création de communautés de chrétiens profondément croyants et convaincus.

\section{Honduras}

En 1955 et 1956, la Société se joint au mouvement d'ensemble et accepte 2 nouveaux territoires de travail en Amérique latine, l'un au Honduras, l'autre au Pérou.

Au Honduras, on confie aux Pères canadiens le territoire de Choluteca d'une population de 120000 habitants avec 5 paroisses, 11 paroisses filiales et de nombreuses dessertes. Alors qu'en 1955, on ne comptait que 3 prêtres dans la région, en 1970, il y en aura 36 dont 28 P.M.E. La population sera passée à 300000 habitants. A la capitale, Tegucigalpa, on trouve 15 canadiens dont 13 P.M.E. dans les 2 paroisses, le petit et le grand séminaire.

Mais les débuts furent difficiles. En arrivant, les prêtres trouvèrent une Église abandonnée de ses fidèles, défigurée et plutôt folklorique. La situation matérielle était lamentable et exigea beaucoup de temps et de travail : chapelles, temples, presbytères, tout était à reconstruire. Les communications presque inexistantes, ainsi que l'éloignement de l'évêque ne favorisaient pas un échange continuel et une pastorale vraiment adéquate. Chacun se débrouillait du mieux qu'il pouvait. De plus, une sacramentalisation à outrance avait pris le dessus sur une véritable évangélisation, et l'on pensait, chez les fidèles que le simple rite suffisait à sauver l'homme. Enfin, le laïc se sentait étranger à l'Église et se considérait plutôt comme un client occasionnel.

Progressivement cependant une stratégie pastorale vit le jour, centrée sur la constitution de communautés de base et sur la promotion humaine. On visa à trouver dans les aldéas des chefs naturels, à les former et à leur laisser prendre de plus en plus de responsabilités et d'initiatives dans leurs communautés locales. Le travail était appuyé et complété par un réseau de près de 700 écoles radiophoniques, par l'organisation de coopératives diverses, de centres communautaires et enfin par un centre d'animation sociale et de la formation de chefs, "La Colmena ». On formait en même temps des catéchètes au nombre de 700 à 800 et enfin on arrivait à constituer près de $\mathbf{2 0 0}$ groupes de célébration de la Parole de Dieu pour les petites communautés nombreuses où le prêtre ne peut se rendre régulièrement. 
Promotion humaine, communautés de base, initiatives communes de développement, évangélisation, qui aboutit à une vraie conscience de son insertion dans l'Église, c'est là l'articulation pastorale suivie au Honduras.

\section{Pérou}

La Société prenait à sa charge une paroisse de 20000 habitants à Lima et le vicariat de Pucallpa dans l'Amazonie avec une population d'environ 60000 habitants et, en 1955, un secteur dans le diocèse d'Ica.

$\mathrm{Au}$ début, les efforts portèrent en dehors du ministère traditionnel, surtout sur l'éducation : c'est l'époque des institutions : écoles primaires, collèges, écoles normales. Parallèlement on fonda de nouvelles paroisses dotées d'un prêtre résident.

On fit aussi des efforts pour le développement socio-économique par la fondation d'une coopérative d'épargne et de crédit (aujourd'hui 5500 membres, la $2^{\circ}$ de tout le pays) puis d'une coopérative d'habitations, une de service téléphonique et une de consommation et de production agricole.

Depuis 5 ans, l'accent porte sur la formation de communautés chrétiennes de base, d'équipes de J.O.C., sur la préparation au mariage, et la participation plus active des fidèles à la vie liturgique. Le but visé est la promotion d'un laïcat chrétien adulte et responsable, intégré et engagé.

Sur le littoral des rivières de la Selva péruvienne qu'on ne peut atteindre que par bateau, se trouvent quelque 150 petits villages de 30 à 70 familles chacun. Au début le ministère là aussi était traditionnel : administration des sacrements. Depuis 3 ou 4 ans, l'accent est mis sur la formation des leaders de ces " caserios * afin d'en faire les animateurs de la communauté humaine et chrétienne. On vise un travail plus en profondeur.

On est ainsi arrivé au Pérou aussi à une pastorale renouvelée avec les caractéristiques suivantes : un plus grand respect des personnes et de leur évolution humaine et chrétienne, le souci de l'éducation au sens des responsabilités, une préoccupation constante plus pour l'animation discrète que pour le dirigisme. Si on ajoute à cela le souci d'un clergé local avec le séminaire-collège de Pucallpa et un effort nouveau et important dans le domaine de la catéchèse, on voit le genre de travail auquel se consacrent 38 P.M.E. dans 11 paroisses.

Les deux dernières missions de la Société acceptées en 1961 se trouvent en Argentine et au Chili. 
Depuis quelques années, le Chili a fait abondamment parlé de lui en raison des transformations politiques et sociales qui s'y produisent. L'Église du Chili depuis le début des années soixante n'a pas été ab= sente de ces efforts de changement. Loin de là, avec des efforts courageux, elle a entrepris sa propre réforme, et elle a décidé de participer le plus possible aux réformes nécessaires d'un pays à la recherche d'une vraie justice sociale.

Les prêtres canadiens ( 27 dont 19 P.M.E. et 8 p.a.m.é.) arrivées au Chili avec une conception pastorale de type paroissial, ont dû bientôt, en face de l'évolution même du pays reviser leur manière de voir. Les efforts portent, de plus en plus sur une pastorale de milieu : plusieurs prêtres se consacrent à des activités apostoliques sur le plan diocésain en tant qu'animateurs : J.O.C., mouvement familial chrétien, etc.

On arrive ainsi à une intégration beaucoup plus grande dans le milieu : intégration au clergé local, et contacts fréquents et intenses avec les gens.

Le résultat de ces transformations c'est une Église où des communautés prennent véritablement vie, où les laïcs sont de plus en plus engagés et dans l'Église et dans le monde et où la communauté eucharistique est de plus en plus vécue par une vraie communauté de personnes.

C'est ce à quoi travaillent les Canadiens et les 40 religieuses canadiennes soit à Santiago, soit à Chillan, soit à Temuco, dans leurs 7 paroisses.

\section{Argentine}

En Argentine, 13 P.M.E., 3 p.a.m.é. et 1 prêtre cubain travaillent depuis 1961 dans 7 paroisses du diocèse de Resistencia, aidés de 12 religieuses canadiennes.

L'Argentine, dont presque toute la population est de souche européenne, est le plus développé de tous les pays latino-américains. Mais, les Européens, surtout Espagnols et Italiens, y ont apporté leurs qualités et leurs défauts : une instruction et une éducation assez avancées, mais aussi un conservatisme qui se manifeste tant au point de vue politique et social que religieux.

Dans ce pays où existe encore l'union de l'Église et de l'État, l'épiscopat est reconnu comme l'un des plus conservateurs du continent. Mais depuis Vatican II, des réformes importantes sont quand même en voie de réalisation.

Dans l'Église locale de Resistencia, les prêtres canadiens cherchent à participer aux nombreuses activités : conseil presbytéral, journée de catéchèse, mouvement familial chrétien, "cursillos de cristiandad ". 
Dans les paroisses, on s'efforce de passer d'une pastorale traditionnelle trop tôt axée sur la sacramentalisation à une pastorale d'ensemble qui, avec l'aide de laïcs engagés, permettra de transformer la religiosité du peuple en un christianisme vécu et incarné, et d'arriver à une vraie évangélisation à travers surtout une catéchèse liturgique renouvelée. Près de 60 catéchètes laïcs dans nos paroisses se consacrent à ce travail.

Dans ce pays se posent aussi de plus en plus des questions politiques et sociales sérieuses. L'Église n'échappe pas à ces nouveaux problèmes : le groupe des prêtres du Tiers-Monde en est un exemple. Elle devra là aussi prendre des positions claires, neuves, si elle veut être de plus on plus présente aux problèmes du peuple chrétien.

\section{Conclusion}

$\mathrm{Si}$ on cherche à caractériser les grandes articulations pastorales des prêtres des Missions-Étrangères durant ces 50 ans d'action, on peut distinguer, en schématisant beaucoup et en grossissant les traits, deux périodes.

Jusque vers les années 60 , leur action a trois pôles principaux :

- prédication de la foi mais qui est encore englobée dans une religion à dominante sacramentelle.

- travail d'éducation au niveau des jeunes par des écoles primaires, secondaires et supérieures, petits et grands séminaires.

- œuvres caritatives : hôpitaux, dispensaires et organisations de type action catholique.

Depuis le début des années 60, il se produit des déplacements d'accents :

- on vise maintenant beaucoup plus à une véritable évangélisation et à constituer de vraies communautés de chrétiens qu'à une sacramentalisation étendue

- le contenu de l'éducation s'est étoffé et a changé de destinataires. On vise beaucoup plus les adultes et on cherche à leur faire prendre conscience de leurs situations concrètes de vie, et à les amener à devenir responsables face à leurs droits et à leurs devoirs à l'intérieur de leurs collectivités propres.

- la nature des œuvres a évolué aussi : coopérative des différents services; écoles radiophoniques; organisations de promotion humaine, formation de chefs.

Si la mission a évolué ces dernières années, on peut affirmer que ce phénomène est loin d'être encore terminé, car les conditions politiques et 
sociales vont changer à une grande rapidité et l'Église devra continuer elle aussi à se transformer.

Ainsi, les conditions de travail ne sont plus les mêmes dans les Églises locales de mission. Celles-ci veulent avec raison prendre en main la direction de leur évolution et exigent ainsi de la part des missionnaires des changements profonds de comportements et d'attitudes.

La nouvelle théologie de la mission, à la suite de Vatican II, invite aussi à une transformation de mentalités et à une modification des points principaux d'action. Il s'agit certes toujours de la prédication de la foi, mais on en arrive à une toute nouvelle articulation entre vie profane et vie chrétienne, entre évangélisation et développement ou révolution, entre Église et Monde.

Tous ces nouveaux courants d'idécs, tous ces changements dans les pays où la Société travaille, conduisent à réévaluer les objectifs de l'action missionnaire et à réétudier nos engagements actuels.

L'évolution rapide actuelle nous amène donc à nous poser de nom. breuses questions sérieuses :

- Qu'est-ce que doit être la mission aujourd'hui ?

- Qu'en est-il du travail dans plusieurs pays actuellement? Doit-on demeurer ou ne vaudrait-il pas mieux partir ?

- Quel doit être l'apostolat en milieu marxiste? Comme à Cuba par exemple?

- Qu'en est-il de l'engagement politique du missionnaire ? Est-il opportun? Quelles devraient être ses formes?

- Comment réaliser la meilleure insertion dans les Églises locales?

- Où devra-t-on faire porter principalement notre action missionnaire dans les années à venir? En Amérique latine, en Afrique, ou en Asie?

Ces problèmes et ces questions rendent la vie missionnaire actuellement plus difficile mais en même temps plus intéressante.

Mais il y a une question à laquelle on a déjà la réponse. C'est celle de la nécessité de la mission, car plus que jamais, l'homme de demain s'interrogera sur lui-même pour se demander: "Qu'est-ce que l'Homme? \$

Et la réponse la plus belle, la plus profonde et la plus étonnante, c'est encore l'Église qui peut l'apporter à cet homme de demain par un témoignage authentique sur le Christ ressuscité.

Claude Guillet, p.m.é. 